\title{
CVA to BPA: Using Balloon Pulmonary Angioplasty to Treat Chronic Thromboembolic Pulmonary Hypertension Post Stroke

\author{
Adinan Kanda, $M D^{1^{*}}$ and Kurt Prins, $M D, P h D^{2}$
} \\ ${ }^{1}$ Internal Medicine Department, University of Minnesota, USA
}

${ }^{2}$ Assistant Professor of Medicine, Cardiovascular Division, Lillehei Heart Institute, University of Minnesota, USA

*Corresponding author: Adinan Kanda, MD, Internal Medicine Department, University of Minnesota, 401 East River Parkway, Minneapolis, MN 55455, USA, Tel: 765-409-6575

\begin{abstract}
In patients with CTEPH who are not eligible for PTE, BPA may be a treatment option. It is important to diagnose and treat patients early since delays are associated with worse clinical outcomes. We present a case of CTEPH where early diagnosis and treatment resulted in normalization of PA pressures.
\end{abstract}

\section{Introduction}

Chronic thromboembolic pulmonary hypertension (CTEPH) is characterized by chronic thromboemboli$\mathrm{sm}$ in the proximal pulmonary arteries and small-vessel disease in the pulmonary capillary system [1]. This results in progressive remodeling of the pulmonary vasculature, increased pulmonary vascular resistance (PVR), pulmonary hypertension, right ventricular remodeling and ultimately right ventricular failure [2]. Early diagnosis of CTEPH is associated with improved outcomes, however, this can be challenging since patients may be asymptomatic for many years or present with non-specific symptoms [3,4]. Pulmonary thrombo endarterectomy (PTE) is the current gold standard treatment for CTEPH butmay not be an option for up to one-third patients. For such patients, balloon pulmonary angioplasty (BPA) has emerged as an efficacious alternative to PEA [5-9]. Here, we report the case of a patient with CTEPH who was diagnosed early and treated successfully with BPA.

\section{Learning Objectives}

- To realize the importance of early diagnosis in CTE$\mathrm{PH}$ given the association with better outcomes.

- To highlight that early diagnosis of CTEPH can be challenging.

- To recognize BPA as a treatment option for patients not eligible for PTE.

\section{Case Presentation}

A 35-year-old male with a history of hereditary spherocytosis with splenectomy fifteen years prior, presented to cardiology clinic for evaluation of patent foramen ovale (PFO), right ventricular enlargement and elevated right-sided pressures observed on echocardiogram. Two months prior, he experienced an acute ischemic stroke, and during the work-up for his CVA an echocardiogram revealed severe pulmonary hypertension with a right ventricular systolic pressure (RVSP) of 80 $\mathrm{mmHg}$. His right ventricle (RV) was dilated, had reduced systolic function, and there was intraventricular septal flattening during systole and diastole consistent with pressure and volume overload. The study also revealed a patent PFO. Transesophageal echocardiography later confirmed the presence of a moderate-sized PFO, which was successfully closed percutaneously with a $25 \mathrm{~mm}$ Gore Cardioform device. Right heart catheterization (RHC) performed at the time of PFO closure showed mean pulmonary artery pressure (mPAP) of 39

Citation: Kanda A, Prins K (2021) CVA to BPA: Using Balloon Pulmonary Angioplasty to Treat Chronic Thromboembolic Pulmonary Hypertension Post Stroke. Int J Clin Cardiol 8:229. doi.org/10.23937/23782951/1410229

Accepted: May 29, 2021: Published: May 31, 2021

Copyright: (c) 2021 Kanda A, et al. This is an open-access article distributed under the terms of the Creative Commons Attribution License, which permits unrestricted use, distribution, and reproduction in any medium, provided the original author and source are credited. 
Table 1: Values from right heart catheterization $(\mathrm{RHC})$ before and after BPA.

\begin{tabular}{|l|l|l|}
\hline & Pre-BPA & Post-BPA \\
\hline Mean Pulmonary Artery Pressure (mPAP) & $31 \mathrm{mmHg}$ & $21 \mathrm{mmHg}$ \\
\hline Pulmonary Capillary Wedge pressure (PCWP) & $17 \mathrm{mmHg}$ & $13 \mathrm{mmHg}$ \\
\hline Pulmonary Vascular Resistance (PVR) & 2.53 Woods Units & 1.16 Woods Units \\
\hline
\end{tabular}

A

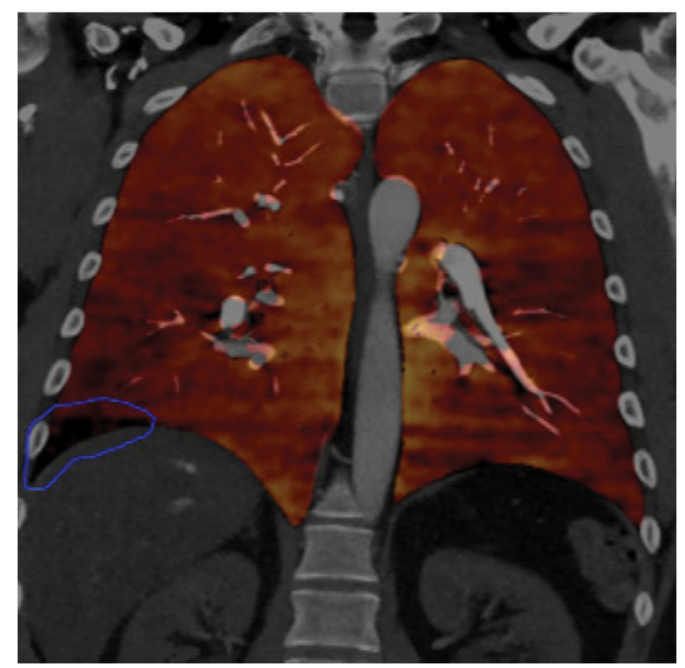

B

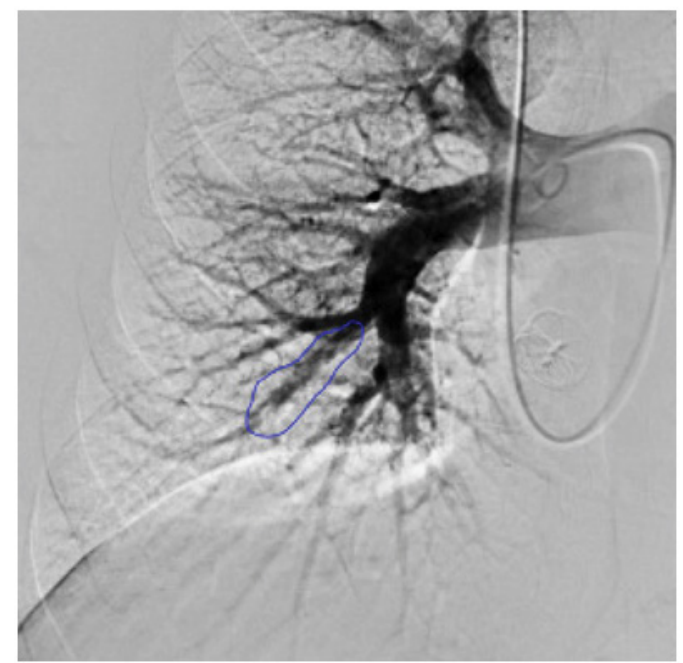

Figure 1: A) CT pulmonary angiogram showing perfusion defect in the right lower lobe (blue outline); B) Invasive pulmonary angiogram showing chronic obstruction of the $A 8$ branch of the right pulmonary artery.

$\mathrm{mmHg}$, pulmonary capillary wedge pressure (PCWP) of $10 \mathrm{mmHg}$ and pulmonary vascular resistance (PVR) of 3 Woods Units. A computed tomography pulmonary angiogram (CTPA) revealed possible isolated subsegmental right lower lobe pulmonary embolus (PE) and a ventilation-perfusion (VQ) scan showed small to moderate-sized mismatched perfusion defects in the right lower lobe superior segment and anterior segment right upper lobe.

He underwent a repeat $\mathrm{RHC}$ about 8 months later, which showed mean pulmonary (mPAP) of $36 \mathrm{mmHg}$, PCWP of $17 \mathrm{mmHg}$ and PVR of 3 Woods Units. VQ scan at that time redemonstrated multiple perfusion defects in the right lung, including the superior segment of the right lower lobe. High-resolution computed tomography (CT) showed no evidence of acute or interstitial lung disease. The patient's cardiologist suspected a diagnosis of chronic thromboembolic pulmonary hypertension (CTEPH) and referred the patient to a pulmonary hypertension $(\mathrm{PH})$ referral center. At the time of referral, the patient had minimal limitations with his exercise capabilities, consistent with World Health Organization (WHO) functional class II.

His initial work-up was remarkable for a normal natriuretic peptide (NT-pro-BNP) concentration of $73 \mathrm{pg} /$ $\mathrm{mL}$. Echocardiography showed mild PH with RVSP of 43 $\mathrm{mmHg}$. LV function and morphology were preserved. CTPA showed a perfusion defect in the right lower lobe (Figure 1A). RHC showed mPAP of $31 \mathrm{mmHg}$, PCWP of
$17 \mathrm{mmHg}$ and PVR of 2.53 Woods Units (Table 1). An invasive pulmonary angiogram revealed chronic obstruction of the A8 branch of the right pulmonary artery with poor filling of distal branches (Figure 1B).

The patient was not a candidate for pulmonary thromboendarterectomy (PTE) since he had an isolated distal lesion. The decision was therefore made to perform a balloon pulmonary angioplasty (BPA) of the A8 branch of the right pulmonary artery. For the procedure, the patient's right femoral vein was accessed under ultrasound guidance without any difficulty. A long 8 French sheath was then placed the right pulmonary artery. Using this sheath, a 6 French JR4 guiding catheter was used to engage the right A10 segment and a weblike lesion in the proximal A10 segment was noted. A Sion blue wire was used to cross the A10 lesion and the lesion was serially dilated using $3.0 \times 15 \mathrm{~mm}, 3.5 \times$ $15 \mathrm{~mm}$ and $4.0 \times 12 \mathrm{~mm}$ emerge balloons. Subsequently, a 6 French multipurpose guide was used to engage the right $A 8$ segment. A Sion blue wire was used to cross the $A 8$ lesion and the lesion was serially dilated using $3.0 \times 15 \mathrm{~mm}, 3.5 \times 15 \mathrm{~mm}$ and $4.0 \times 12 \mathrm{~mm}$ emerge balloons with good results (Figure $2 \mathrm{~A}$, Figure $2 \mathrm{~B}$, Figure $2 \mathrm{C}$ and Figure 2D). There were no periprocedural complications.

RHC performed one month after BPA showed MPAP of $21 \mathrm{mmHg}$ (decreased from $31 \mathrm{mmHg}$ ), PCWP of 13 $\mathrm{mmHg}$ (decreased from $17 \mathrm{mmHg}$ ) and PVR of 1.16 Woods Units (decreased from 2.53 Woods Units) (Table 1). 
A

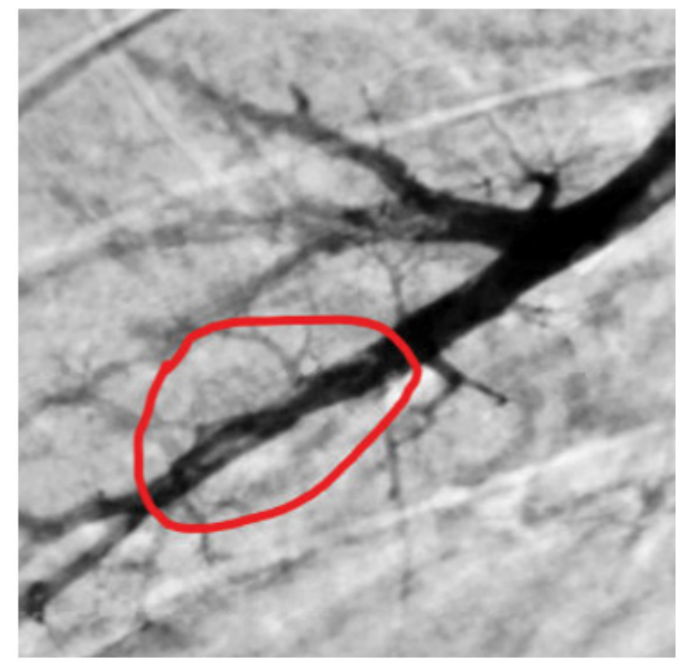

C

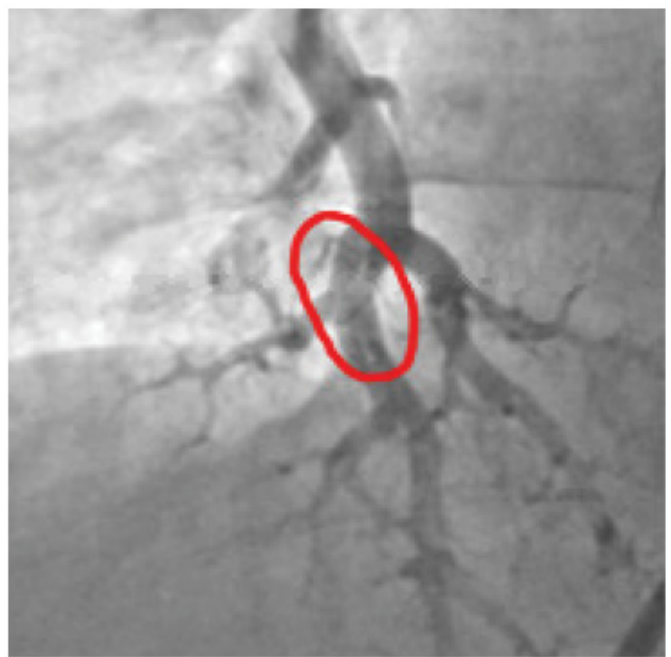

B

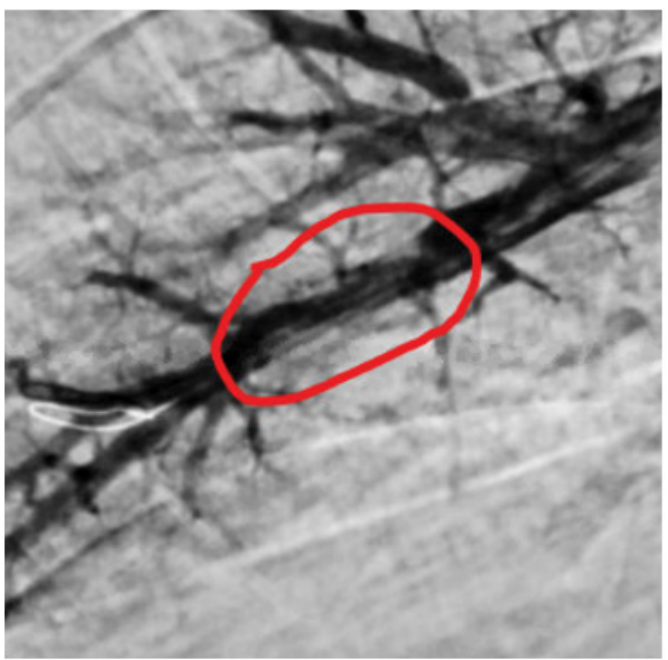

D

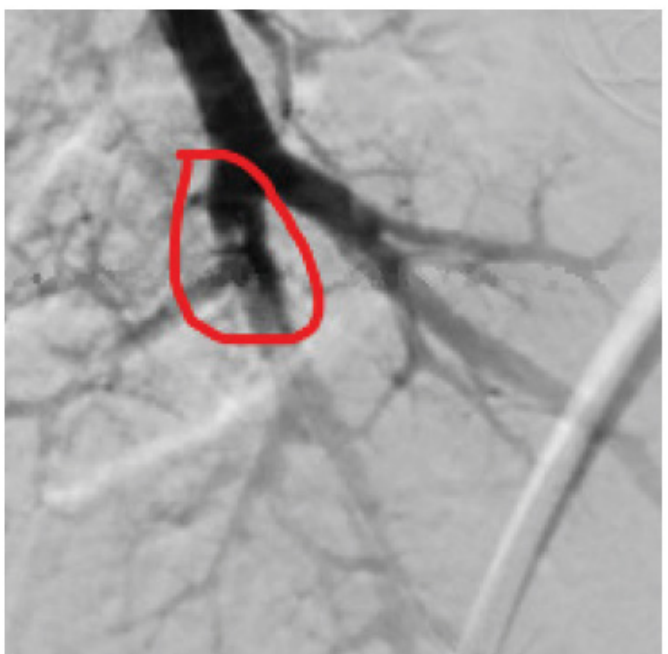

Figure 2: A) A8 segmental pulmonary artery pre-BPA and B) Post-BPA; C) A10 segmental pulmonary artery pre-BPA and D) Post-BPA.

Echocardiography showed normal global RV and LV function. Patient is now WHO functional class 1.

\section{Discussion}

CTEPH, classified within group $4 \mathrm{PH}$, is characterized by chronic thromboembolism in proximal pulmonary arteries and small-vessel disease involving both the pulmonary venous and pulmonary arterial capillary systems [1]. These changes result in progressive remodeling of the pulmonary vasculature, increased PVR, pulmonary hypertension, RV remodeling and ultimately RV failure [2]. CTEPH should be considered in the evaluation of patients with pulmonary hypertension since it is treatable and potentially curable [8]. It is especially important to diagnosis and treat CTEPH earlier in the course of the disease, as delays in treatment have been associated with worse outcomes [4]. Making an early diagnosis of CTE$\mathrm{PH}$, however, can be challenging since patients may be asymptomatic for many years or present with non-spe- cific symptoms [3]. The presence of thromboemboli on CTPA and mismatched perfusion defects on VQ scan in a patient who has received at least 3 months of therapeutic anticoagulation is diagnostic of CTEPH [5].

PTE is the current gold standard treatment for CTE$\mathrm{PH}$, however, about a third of patients may be deemed ineligible due to high-risk comorbidities, persistent pulmonary hypertension post-PTE or presence of chronic thromboembolism in the distal pulmonary arteries [69]. For such patients, BPA has emerged as an efficacious alternative to PTE, and has been reported to improve symptoms, hemodynamics, exercise capacity and right ventricular function when performed at an expert medical center $[6,7]$. In a meta-analysis by Kalra, et al. which included a total of 1604 patients (755 patients with inoperable CTEPH treated with BPA; 849 patients with inoperable CTEPH treated with pulmonary vasodilators) across 34 studies, BPA was associated with greater im- 
provement in 6-minute walk distance, PVR and mPAP compared to pulmonary vasodilator therapy. BPA was notably associated with more complications due to its invasive nature [9].

As discussed above, CTEPH can be difficult to diagnose earlier in the course of the disease. The patient in this case was diagnosed at an early stage as part of the work-up for his CVA, which portends better outcomes. He had no prior history of $\mathrm{PE}$, similar to $21.2 \%$ of patients with CTEPH in a study by Martinez, et al. who were found to have no history of PE [10]. Interestingly, this patient had a history of splenectomy, which is a known risk factor for CTEPH [1]. He was not a candidate for PTE since his initial evaluation showed an isolated distal lesion. He, instead, underwent BPA which normalized his PA pressures.

\section{References}

1. (2020) Group 4 Pulmonary Hypertension - Clinical Key.

2. Delcroix M, Noordegraaf AV, Fadel E, Lang I, Simonneau G, et al. (2013) Vascular and right ventricular remodelling in chronic thromboembolic pulmonary hypertension. Eur Respir J 41: 224-232.

3. Nishiyama KH, Saboo SS, Tanabe $\mathrm{Y}$, Jasinowodolinski D, Landay MJ, et al. (2018) Chronic pulmonary embolism: Diagnosis. Cardiovasc Diagn Ther 8: 253-271.
4. Held M, Hesse A, Gött F, Holl R, Hubner G, et al. (2014) A symptom-related monitoring program following pulmonary embolism for the early detection of CTEPH: A prospective observational registry study. BMC Pulm Med 14: 141.

5. Memon HA, Lin CH, Guha A (2016) Chronic thromboembolic pulmonary hypertension: Pearls and Pitfalls of Diagnosis. Methodist DeBakey Cardiovasc J 12: 199-204.

6. Ogo T (2015) Balloon pulmonary angioplasty for inoperable chronic thromboembolic pulmonary hypertension. Curr Opin Pulm Med 21: 425-431.

7. Kim NH, Delcroix M, Jais $\mathrm{X}$, Madani MM, Matsubara $\mathrm{H}$, et al. (2019) Chronic thromboembolic pulmonary hypertension. Eur Respir J 53: 1801915.

8. Kim NH, Delcroix M, Jenkins DP, Channick R, Dartevelle P, et al. (2013) Chronic thromboembolic pulmonary hypertension. J Am Coll Cardiol 62: D92-D99.

9. Kalra R, Duval S, Thenappan T, Raveendran G, Pritzker M, et al. (2020) Comparison of balloon pulmonary angioplasty and pulmonary vasodilators for inoperable chronic thromboembolic pulmonary hypertension: A systematic review and meta-analysis. Sci Rep 10: 8870.

10. Martinez C, Wallenhorst C, Teal S, Cohen AT, Peacock AJ (2018) Incidence and risk factors of chronic thromboembolic pulmonary hypertension following venous thromboembolism, a population-based cohort study in England. Pulm Circ 8: 2045894018791358. 